\title{
A Bibliometric Analysis of European versus USA Research in the Field of Addiction. Research on Alcohol, Narcotics, Prescription Drug Abuse, Tobacco and Steroids 2001-2011
}

\author{
Jørgen G. Bramness ${ }^{a, b}$ Beate Henriksen ${ }^{c}$ Olle Person ${ }^{d}$ Karl Mann ${ }^{e, f}$ \\ ${ }^{a}$ Norwegian Centre for Addiction Research, University of Oslo, b Department of Pharmacoepidemiology, \\ Norwegian Institute of Public Health, and 'Norwegian Institute for Alcohol and Drug Research, Oslo, Norway; \\ d Information Science Unit, Department of Sociology, Umeå University, Umeå, Sweden; ${ }^{\circ}$ Central Institute of \\ Mental Health, Medical Faculty Mannheim, University Heidelberg, and ${ }^{\mathrm{f} E u r o p e a n ~ F e d e r a t i o n ~ o f ~ A d d i c t i o n ~ S o c i e t i e s, ~}$ \\ Mannheim, Germany
}

Key Words

Abuse and dependence · European versus USA research

Abstract

Background: To compare the publication and citation rate within the areas of drug abuse and dependence research in Europe with that in the USA. Methods: This is a bibliometric study using the Thomson Reuters Web of Knowledge as data source, 40 key words were used as search terms, but certain scientific publications not concerning the issue were excluded. Scientific publications from Denmark, England, Finland, France, Germany, Italy, the Netherlands, Norway, Spain, Sweden, and the USA were studied. The number of publications in each country and in each year in addition to the citation indices for these publications was retrieved. $\boldsymbol{R e}$ sults: Approximately two thirds of the publications came from the USA. Both in absolute and relative figures, Europe lagged behind. The trend over the last decade was a greater gap between the amount of research performed in Europe versus the USA. There were thematic differences. Smaller European countries had a greater relative publication rate. The citations were relatively evenly distributed. Conclu- sions: It has been claimed that $85 \%$ of the world's research within the field of drug abuse and dependence is carried out in the USA. This study challenges this figure, but European research within this field is lagging behind.

Copyright $\odot 2013$ S. Karger AG, Basel

\section{Introduction}

Bibliometric analysis may used as a tool to evaluate research within a specific field [1]. By looking at the number of papers and how often these are cited it is possible to have a surrogate or proxy measure of the quantity and quality of the research within that field. There are obvious shortcomings to the bibliometric methods with low sensitivity, possibly also low specificity and unknown external validity. No database of scientific research will cover all publications and some fields may suffer more than others. And not all articles found by this method are relevant. Furthermore, the number of times a paper is cited may not reflect its scientific or societal value $[2,3]$. However, for comparisons over time or between institutions or countries, these objections are less important and bib-

\section{KARGER}

E-Mail karger@karger.com

www.karger.com/ear
(C) 2013 S. Karger AG, Basel

1022-6877/14/0201-0016\$39.50/0
Prof. Jørgen G. Bramness, MD, PhD

Norwegian Centre for Addiction Research (SERAF)

Institute of Psychiatry, University of Oslo

Kirkeveien 166, NO-0407 Oslo (Norway)

E-Mail j.g.bramness@medisin.uio.no 
liometric analysis may be of value, because the shortcomings will have a similar impact across different settings.

Traditionally, a large share of addiction research in the world has been performed in the USA. The National Institute of Drug Abuse (NIDA) has a budget of USD 1 billion each year to finance intra- and extramural research [4]. This is, of course, a very large contribution and is of great global value. It gives NIDA a leading role in drug research worldwide, funding by their own estimates as much as $85 \%$ of the world's research within the field [5]. In addition The National Institute on Alcohol Abuse and Alcoholism (NIAAA) has a yearly budget of USD 0.5 billion, increasing the importance of US funding. The knowledge this research may, of course, be applicable outside the USA. This would be especially true for basic research, but coming to clinical research it may, in certain situations, be more difficult to adopt findings from the USA to Europe [6]. European countries need to do their own research. Earlier surveys covering the period up to 2000 have shown that even if the amount of European research in the field is increasing, it is still substantially smaller than in the USA [7]. The present study follows up on this finding to investigate what has happened during the last decade.

Research is very important because we need a clear evidence base for treatment [8]. Only through the application of evidence-based treatments can we offer our patients good treatment and use our society's resources optimally [9]. This is true for the field of addiction as for other fields of healthcare, and scientific research has brought new knowledge to the field of addiction during recent years [10]. Scientifically trained professionals are, furthermore, less prone to base their treatment choices on ideologies or political beliefs and may be less vulnerable to commercial impact. This may be an especially important point for the area of alcohol and drug abuse and addiction treatment.

It should thus be almost self-evident that research within the areas of drug abuse and dependence and their treatments is of crucial importance. The World Health Organization estimates that each year the harmful use of alcohol results in 2.5 million deaths [11]. Globally, every year more than 300,000 young people between the ages of 15 and 29 die from alcohol-related causes, resulting in 9\% of all deaths in that age group. It has been estimated that alcohol use disorder is the one of the most important causes of lost disability-adjusted life-years in Europe [12], imposing an enormous economic burden $[13,14]$. Worldwide, as many as 200 million people use illicit drugs every year, equalling the burden of disease from this drug group with what we see following the use of alcohol [15].
In Europe more than 15 million people are estimated to have alcohol or drug dependence [12]. These figures make drug abuse one of the most significant health issues and one of the greatest social challenges of our time.

The aim of this study was to perform a bibliometric study in the field of addiction in 10 European countries and compare these with the same type of publication data from the USA in the years 2001-2011 (see online suppl. table, www.karger.com/doi/10.1159/348260).

\section{Materials and Methods}

The survey began with a key word search to identify as many relevant research papers between January 2001 and December 2011 as possible using the database of published scientific journals maintained by Thomson Reuters (formerly ISI) Web of Knowledge. The 40 key words and sorting criteria used are specified in appendix 1. We repeated the search for the following countries in the whole period: Denmark, England, Finland, France, Germany, Italy, the Netherlands, Norway, Spain, Sweden, and the USA. These countries were selected because they were part of an earlier Scandinavian survey (NAS), adding some countries identified to have the largest share of addiction research in an earlier publication [12].

A multidisciplinary field, such as this, represents special challenges in a survey. Although there is a set of core journals, a great number of articles are scattered over a vast number of disciplines, and relevant papers may be published in general journals in a variety of fields. Further, many key words, such as alcohol, are used in a variety of natural science papers which are not relevant. Attempts were made to exclude these papers from the analysis (see appendix 1 and online supplement table). To increase accuracy the search was restricted to the scientific paper topic field, requiring that at least one key word was present indicating an alcohol, narcotics, prescription drugs and tobacco (ANDT) substance.

For some field-specific analyses, the drugs were grouped according to the following system: alcohol: alcohol, beer, drink, ethanol, liquor, wine; illicit drugs: buprenorphine, amphetamine, cannabinol, cannabis, cocaine, codeine, heroin, LSD, mescaline, methadone, methamphetamine, morphine, narco, opiate, opioid, PCP, THC; medicinal drugs: amphetamine, barbiturates, benzodiazepines, buprenorphine, codeine, methadone, morphine, opiate, opioid, prescri, tramadol; steroids: steroid and tobacco: nicotine, smok, snuff, tobac.

In the present study we looked at the number of papers (per year, country and drug group), the relative number of papers (per million inhabitants in the different countries), and the trends over time for this measure. We also studied the number of citations per paper for each year per year and on average.

\section{Results}

In absolute figures, the USA published far more papers during the study period than any European country (table 1) and than Europe as a whole. Even if some 
Fig. 1. Relative number of publications (per million inhabitants) within drug abuse research in 10 European countries and the USA for the period 2001-2011.

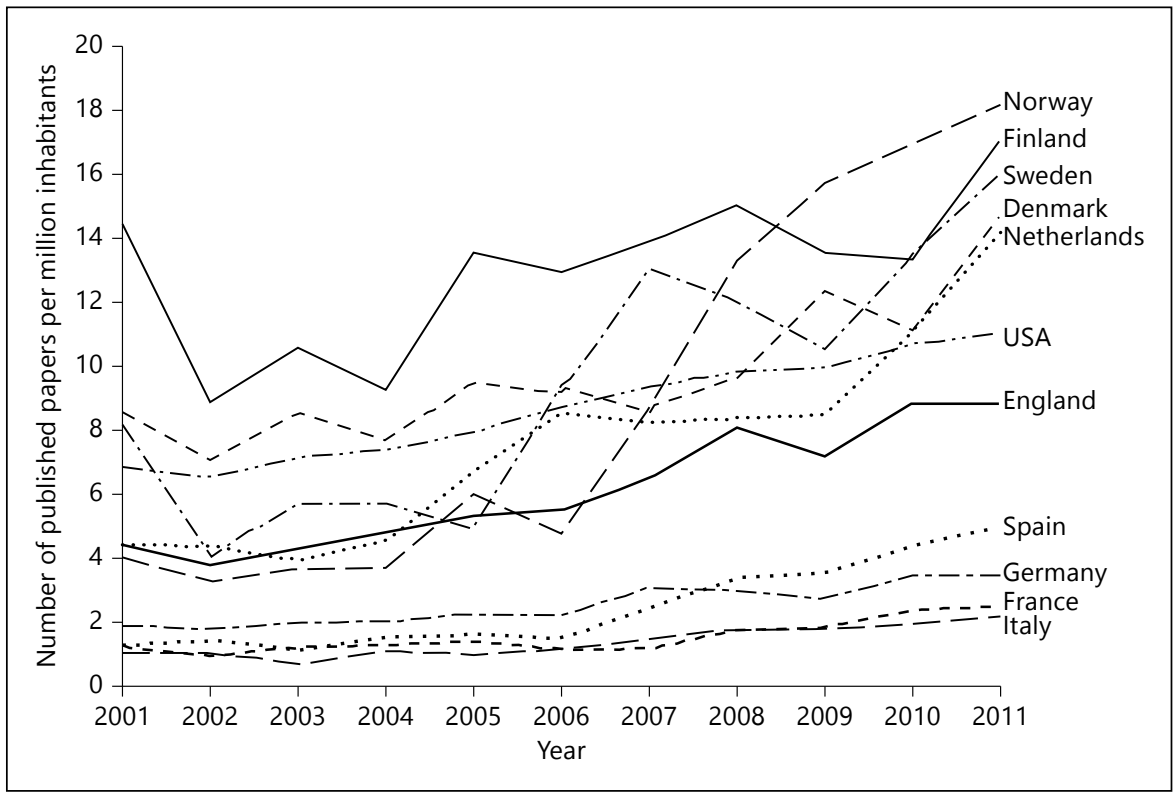

Table 1. Absolute number of papers and relative number of papers per million inhabitants in 10 European countries and the USA in the field of addiction research 2001-2011

\begin{tabular}{|c|c|c|c|c|}
\hline & \multicolumn{2}{|c|}{ Absolute number of papers } & \multicolumn{2}{|c|}{ Relative number of papers } \\
\hline England & 3,368 & $26.7(20.5-32.9)$ & 67.4 & $0.53(0.41-0.66)$ \\
\hline Finland & 769 & $2.5(0.2-4.9)$ & 142.4 & $0.47(0.03-0.90)$ \\
\hline Italy & 903 & $7.8(5.0-10.6)$ & 15.1 & $0.13(0.08-0.18)$ \\
\hline Netherlands & 1,407 & $15.2(10.6-19.8)$ & 82.8 & $0.89(0.63-1.16)$ \\
\hline Norway & 542 & $6.7(4.0-9.3)$ & 110.6 & $1.37(0.83-1.91)$ \\
\hline Spain & 1,245 & $17.6(12.1-23.1)$ & 27.1 & $0.38(0.26-0.50)$ \\
\hline Sweden & 1,097 & $7.0(4.1-9.9)$ & 115.5 & $0.74(0.43-1.05)$ \\
\hline 10 European countries sum & 13,109 & $112.8(85.8-139.8)$ & 38.2 & $0.33(0.25-0.41)$ \\
\hline
\end{tabular}

smaller Northern European countries (Norway, Finland, Sweden, Denmark and the Netherlands) had a relatively high publishing rate (fig. 1; table 1), Europe as a whole published relatively less than the USA with 13,109 papers on a population basis of 343 million inhabitants in the studied countries, compared to 28,211 papers on a population basis of 296 million inhabitants in the USA.

The absolute increase in publications was larger in the USA (an increase on average of 141.0 papers per year;
95\% CI 123.4-158.5) than in any single European country (table 1). Europe as a whole had an average increase of 113.8 papers per year (95\% CI 86.3-141.2). The USA is thus gaining on Europe in this field. The relative increase in the number of published papers (per million inhabitants) was greater in Northern Europe - Norway, Denmark, the Netherlands and Sweden (table 1), but considering the small sizes of these countries, not enough to allow Europe to compete with the USA. 
Fig. 2. Relative number of publications (per million inhabitants) within 5 different fields concerning drug abuse research in 10 European countries and the USA for the period 2001-2011.

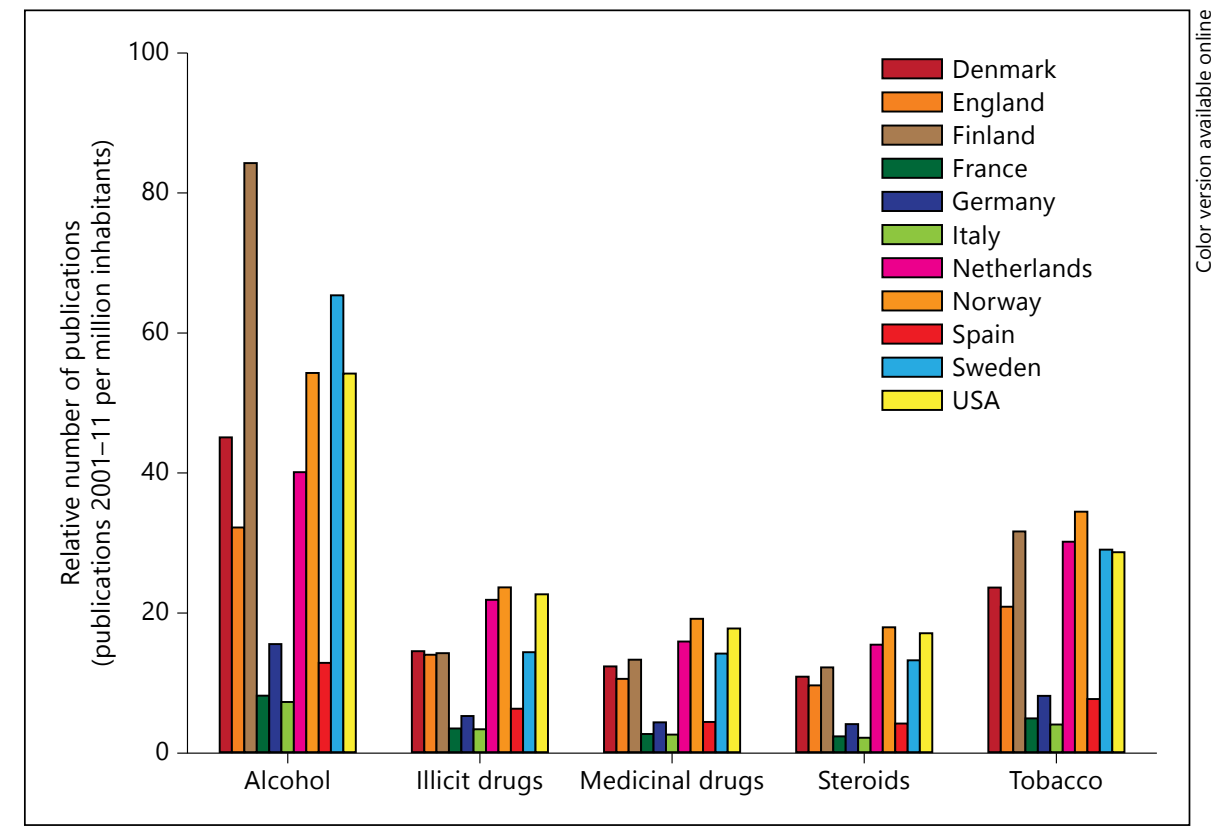

Table 2. Number of scientific papers from each country studied (2001-2011) divided into subject fields

\begin{tabular}{|c|c|c|c|c|c|}
\hline & Alcohol & Illicit drugs & Medicinal drugs & Steroids & Tobacco \\
\hline Denmark & $248(55.1)$ & $80(17.8)$ & $68(15.1)$ & $60(13.3)$ & $130(28.9)$ \\
\hline England & $1,611(47.8)$ & $702(20.8)$ & $530(15.7)$ & $484(14.4)$ & $1,045(31.0)$ \\
\hline France & $517(49.0)$ & $222(21.0)$ & $171(16.2)$ & $150(14.2)$ & $312(29.6)$ \\
\hline Germany & $1,276(56.1)$ & $434(19.1)$ & $359(15.8)$ & $340(15.0)$ & $671(29.5)$ \\
\hline Italy & $438(48.5)$ & $204(22.6)$ & $158(17.5)$ & $132(14.6)$ & $245(27.1)$ \\
\hline Spain & $593(47.6)$ & $292(23.5)$ & $204(16.4)$ & $194(15.6)$ & $355(28.5)$ \\
\hline Sweden & $621(56.6)$ & $137(12.5)$ & $135(12.3)$ & $126(11.5)$ & $276(25.2)$ \\
\hline USA & $16,042(56.9)$ & $6,713(23.8)$ & $5,264(18.7)$ & $5,064(18.0)$ & $8,490(30.1)$ \\
\hline
\end{tabular}

The percentage of papers covering this specific area within each country is shown in parentheses. The total of the percentages is more than 100 as many papers are about more than one substance.

The number of papers in each subject area is given in table 2. The USA had, of course, the greatest number of publications in all these fields. Many papers cover more than one subject area so the number of papers in table 2 adds up to more than the total number of published papers (table 1). There were more papers covering several fields from the USA and the Netherlands, resulting in them having a large share of their papers in all the different fields. Figure 2 gives the relative number of papers in each field (per million inhabitants) showing that in the field of alcohol research Finland and Sweden were leading. In the fields of illicit drugs, medicinal drug abuse and steroids Norway had the highest relative number of publications, followed by the USA. In the tobacco field, Norway also had the highest relative number of publications followed by Finland.

Figure 3 shows the average number of citations per year in the years 2001-2011 for papers published in the years 2001-2011. The Netherlands had the highest citation rate followed by the USA and Denmark. Norway, 
Fig. 3. Average number of citations per year in the years 2001-2011 for papers published $2001-2011$ by country.

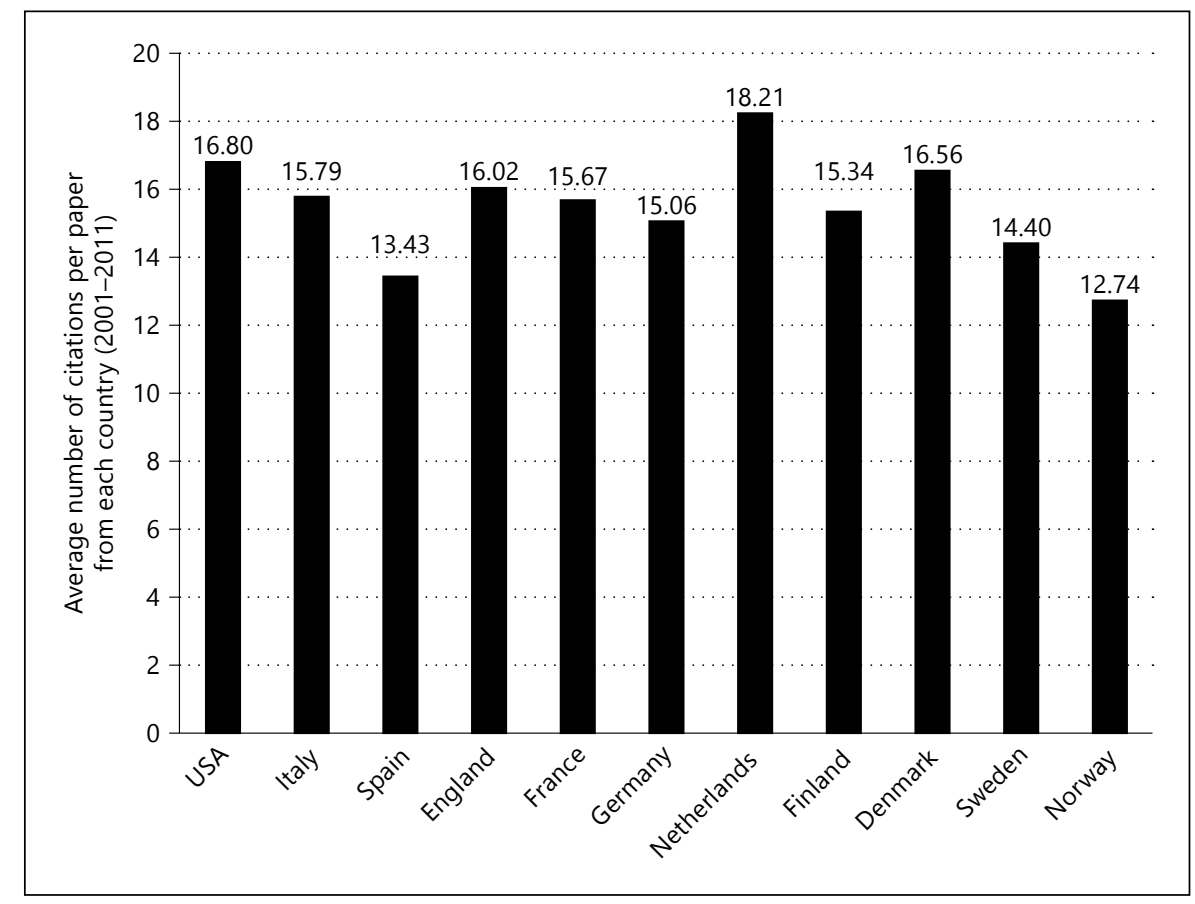

Spain and Sweden had the lowest citation rates per paper in the period with $70-80 \%$ of the citations compared to the citation rate in the Netherlands.

\section{Discussion}

This investigation shows that the USA produces far more scientific papers than Europe in the important fields of alcohol and drug abuse and addiction. Even if some smaller European countries reach the same relative publication rate as the USA, Europe is generally lagging behind. Despite Europe's higher population, the absolute number of papers coming from Europe does not reach more than half that published in the USA.

However, we do not find support for the notion that $85 \%$ of all research in the drug abuse field comes from the USA [5]. We have counted all papers from the USA, and only a proportion of the publications from outside the USA (10 European countries), but still find that only two thirds of the papers come from groups in the USA. The number would probably be closer to $50 \%$ if the rest of the world was included. It could be argued that NIDA and NIAAA finance studies abroad, but this is probably not true to a level to bring the figure up to $85 \%$. When we look at citation indices, we see that there are no big differences between countries, so it is also difficult to claim that the
USA is financing $85 \%$ of the research even if some quality marker was added ('good research').

It is striking that many of the smaller European countries (Norway, Finland, Sweden, Denmark and the Netherlands), relative to population size, publish more than both the USA and the larger European countries in this field. For France and Germany and partially for Italy and Spain, it can be argued that they publish papers in languages other than English. A survey from 2005 showed around $21 \%$ of European Union papers within this field were published in languages other than English [7], most often Spanish, French, German, Dutch and Italian. This figure indicates that publications in languages other than English cannot fully explain the relatively low publication rates from France, Germany, Italy and Spain. Even adding $21 \%$ (which would be the total of all non-English papers) to the publication rates would not bring these larger European countries up to the rates of the smaller European countries or the USA. A low publication rate can thus not be explained by publications in local languages. The smaller northern European countries that also publish papers in their own language demonstrate higher publication rates than England, which even publishes even 'local' results in English language journals.

The higher publication rates in the smaller northern European countries are interesting but difficult to explain. Could it be a difference between wine-producing 
and non-producing countries? Could it be for historical reasons, even if Norway has moved from a lower to a higher publication rate? It could be other things like moral, politics or religion, but this is difficult to decide.

For all countries there has been an increase in the number of publications over the period observed (higher increase in the smaller countries mentioned above). This increase is a continuation of a trend observed earlier in a paper covering the time period 1976-2000 [7], and is of course welcomed. This rate is probably influenced by a general increase in publications in science [16]. On average, the publication rate increased less in Europe than in the USA. The USA produced 1,365 more papers than the investigated European countries in 2011. At the current rate this figure will have increased by almost $20 \%$ in 10 years.

When dividing the publications into topics, we see that both the absolute and the relative number of papers to a very large extent follow the trends found for the total number of papers. The exceptions were the Netherlands and the USA which seem to have a lot of papers that deal with many subjects, thus giving them many publications in many fields. Smaller countries like Norway, Sweden and Finland do top the relative rankings in some fields.

Citations can be viewed as a proxy for scientific quality, even if this has been challenged [2,7]. The USA and the Netherlands seem to have the highest rates of citations. Even if citation rates give an impression of the quality and impact of a paper, these rates are biased to publication rate, as authors have a tendency to cite their own papers, and the papers of colleagues they have met or heard. This produces higher citation rates for higher producing scientific groups or countries, and this is maybe also reflected in the citation rates observed, with high producing countries like the USA and the Netherlands demonstrating higher citation rates. Still there are some differences between the countries, the lower countries (Norway, Spain and Sweden) having 20$30 \%$ lower citation rates than the high countries, that may be difficult to explain by other factors than possibly lower scientific value. However it may be difficult to decide if a variation of $20-30 \%$ in the average number of citations is a large figure. Considering the arguments against the citation indices, it may be impossible to conclude that there were large variations in the quality of research between countries.

There are several obvious limitations to our investigation. Firstly we have only investigated publications from 10 European countries. There may be important research from other European countries. We do, however, cover the largest countries in terms of research output. It was documented earlier that these countries probably produce as much as $95 \%$ of the publications from Europe [3]. Secondly we have only investigated one database, and so may not have picked up all publications. The Thomson Reuters Web of Knowledge is, however, known to cover a substantial number of published scientific papers, avoiding too many conference abstracts and the like. Thirdly, we are of course limited by our search terms, which may or may not include or exclude relevant research. In order to really be sure of the inclusions a manual review would be necessary, but the sheer number makes this impossible. Lastly, these database searches have a tendency to leave out books and similar publications, giving a bias towards quantitative research. For the last three limitations there is however no reason to believe that there is a selective bias or discrimination of one particular country, so this should limit the impact of these exclusions on our results.

\section{Conclusion}

The current study does not find support for $85 \%$ of the world's research within the field of alcohol and drug abuse and addiction being done through US funding, but still Europe is lagging behind in research within the field and the gap is increasing. The need for local research is documented [6]. We cannot afford to be left behind in a field of such great importance.

\section{Disclosure Statement}

The authors have no conflicts of interest to disclose.

\section{Appendix 1}

The Thomson Reuters (formerly ISI) Web of Knowledge was searched during May and June 2012 for the period January 2001 and December 2011 for the countries Denmark, England, Finland, France, Germany, Italy, the Netherlands, Norway, Spain, Sweden, and the USA.

The key words and sorting criteria were as follows:

In the title, the following terms were searched for 'drug abuse*', 'drug use*', 'substance abuse*', 'substance use*', abstinen*, addict*, alcohol*, amphetamine*, barbiturates*, beer*, benzodiazepines*, buprenorphine*, cannabinol*, cannabis*, cocaine*, codeine*, drink*, ecstasy*, ethanol*, heroin*, liquor*, LSD*, MDA*, MDMA*, mescaline*, methadone*, methamphetamine*, morphine*, narco*, nicotine*, opiate*, opioid*, PCP*, prescription drugs* ${ }^{*}$, smok $^{*}$, snuff*, THC*, tobac*, tramadol* or wine*, where 
$\left.{ }^{*}\right)$ depicts any following term. Inverted column holds words together in accurate phrasing. The search was limited to scientific articles. Articles from the following journal categories were excluded: acoustics or agricultural engineering or agriculture; dairy and animal science or agriculture; multidisciplinary or astronomy and astrophysics or automation and control systems or biochemical research methods or biochemistry and molecular biology or biodiversity conservation or biology or biophysics or biotechnology and applied microbiology or chemistry; analytical or chemistry; applied or chemistry; inorganic and nuclear or chemistry; medicinal or chemistry; multidisciplinary or chemistry; organic or chemistry; physical or computer science; artificial intelligence or computer science; hardware and architecture or computer science; information systems or computer science; interdisciplinary applications or computer science; software engineering or computer science; theory and methods or construction and building technology or energy and fuels or engineering; aerospace or engineering; biomedical or engineering; chemical or engineering; civil or engineering; electrical and electronic or engineering; environmental or engineering; industrial or engineering; manufacturing or engineering; mechanical or engineering; multidisciplinary or entomology or forestry or geography; physical or geology or geosciences; multidisciplinary or marine and freshwater biology or materials science; biomaterials or materials science; ceramics or materials science; characterization and testing or materials science; coatings and films or materials science; composites or materials science; multidisciplinary or materials science; paper and wood or materials science; textiles or mathematical and computational biology or mathematics or mathematics; applied or mathematics; interdisciplinary applications or mechanics or metallurgy and metallurgical engineering or meteorology and atmospheric sciences or nanoscience and nanotechnology or nuclear science and technology or operations research and management science or optics or physics; applied or physics; atomic; molecular and chemical or physics; condensed matter or physics; fluids and plasmas or physics; mathematical or physics; multidisciplinary or physics; nuclear or physics; particles and fields or polymer science or remote sensing or robotics or soil science or thermodynamics or water resources.

\section{References}

1 Moed H, Burger J, Frankfort J, van Raan A: The use of bibliometric data for the measurement of university research performance. Res Policy 1985;14:131-149.

$\checkmark 2$ Valderrama Zurian JC, Aleixander R, Castellano M: Citation count analysis in addiction (2001). Addiction 2004;99:387-388.

- 3 West R, Mcllwaine A: What do citation counts count for in the field of addiction? An empirical evaluation of citation counts and their link with peer ratings of quality. Addiction 2002;97:501-504.

4 NIDA: Fiscal Year 2012 Budget Information - Congressional Justification for National Institute on Drug Abuse. June 5, 2012.

5 NIDA: NIDA - National Institute of Drug Abuse. June 5, 2012.

6 Mann K: Why should we need a European Federation of Addiction Societies? Addiction 2012;107:692-693.
Sanchez-Carbonell X, Guardiola E, Belles A, Beranuy M: European Union scientific production on alcohol and drug misuse (19762000). Addiction 2005;100:1166-1174.

$\checkmark 8$ Carroll KM: Dissemination of evidencebased practices: how far we've come, and how much further we've got to go. Addiction 2012; 107:1031-1033.

9 Donaldson C, Mugford M, Vale L: EvidenceBased Health Economics: From Effectiveness to Efficiency in Systematic Reviews. London, BMJ Books, 2002.

10 Sellman D: The 10 most important things known about addiction. Addiction 2010;105: 6-13.

11 WHO: The Global Burden of Disease: 2004 Update. Geneva, WHO, 2008.

12 Wittchen HU, Jacobi F, Rehm J, Gustavsson A, Svensson M, Jonsson B, et al: The size and burden of mental disorders and other disorders of the brain in Europe 2010. Eur Neuropsychopharmacol 2011;21:655-679.
3 Effertz T, Mann K: The burden and cost of disorders of the brain in Europe with the inclusion of harmful alcohol use and nicotine addiction. Eur Neuropsychopharmacol 2012 (E-pub ahead of print).

14 Gustavsson A, Svensson M, Jacobi F, Allgulander C, Alonso J, Beghi E, et al: Cost of disorders of the brain in Europe 2010. Eur Neuropsychopharmacol 2011;21:718779.

15 Degenhardt L, Hall W: Extent of illicit drug use and dependence, and their contribution to the global burden of disease. Lancet 2012; 379:55-70.

16 Jones AW: The distribution of forensic journals, reflections on authorship practices, peer review and role of the impact factor. Forensic Sci Int 2007;165:115-128. 